Research Paper

\title{
Synthesis and Evaluation of BMMSC-seeded BMP- 6/nHAG/GMS Scaffolds for Bone Regeneration
}

\author{
Xuewen $\mathrm{Li}^{1}$, Ran Zhang2, Xuexin Tan², Bo Li1, Yao Liu ${ }^{3}$, Xukai Wang ${ }^{2}$ \\ 1. Department of Oral Anatomy and Physiology, School of Stomatology, China Medical University, Shenyang, China \\ 2. Department of Oral and Maxillofacial Surgery, School of Stomatology, China Medical University, Shenyang, China \\ 3. Department of Pediatric Dentistry, School of Stomatology, China Medical University, Shenyang, China \\ $\triangle$ Corresponding author: Dr. Prof. Xukai Wang. Department of Oral and Maxillofacial Surgery, School of Stomatology, China Medical University, 117 Nanjing \\ North Street, Shenyang, 110002, China. Telephone number: +862431927862; E-mail: wangxukai1518@hotmail.com \\ (C) Ivyspring International Publisher. This is an open access article distributed under the terms of the Creative Commons Attribution (CC BY-NC) license \\ (https://creativecommons.org/licenses/by-nc/4.0/). See http://ivyspring.com/terms for full terms and conditions.
}

Received: 2018.12.03; Accepted: 2019.05.11; Published: 2019.06.10

\begin{abstract}
Bioactive scaffolding materials and efficient osteoinductive factors are key factors for bone tissue engineering. The present study aimed to mimic the natural bone repair process using an osteoinductive bone morphogenetic protein (BMP)-6-loaded nano-hydroxyapatite (nHA)/gelatin (Gel)/gelatin microsphere (GMS) scaffold pre-seeded with bone marrow mesenchymal stem cells (BMMSCs). BMP-6-loaded GMSs were prepared by cross-linking and BMP-6/nHAG/GMS scaffolds were fabricated by a combination of blending and freeze-drying techniques. Scanning electron microscopy, confocal laser scanning microscopy, and CCK-8 assays were carried out to determine the biocompatibility of the composite scaffolds in vitro. Alkaline phosphatase (ALP) activity was measured to evaluate the osteoinductivity of the composite scaffolds. For in vivo examination, critical-sized calvarial bone defects in Sprague-Dawley rats were randomly implanted with BMMSC/nHAG/GMS and BMMSC/BMP-6/nHAG/GMS scaffolds, and compared with a control group with untreated empty defects. The BMP-6-loaded scaffolds showed cytocompatibility by favoring BMMSC attachment, proliferation, and osteogenic differentiation. In radiological and histological analyses, the BMMSC-seeded scaffolds, especially the BMMSC-seeded BMP-6/nHAG/GMS scaffolds, significantly accelerated new bone formation. It is concluded that the BMP-6/nHAG/GMS scaffold possesses excellent biocompatibility and good osteogenic induction activity in vitro and in vivo, and could be an ideal bioactive substitute for bone tissue engineering.
\end{abstract}

Key words: Osteoconductive scaffold, bone marrow mesenchymal stem cells, bone morphogenetic protein- 6 , bone tissue engineering

\section{Introduction}

Bone, which is crucial for physiological functions, can be impaired in situations that involve trauma, pathological disease, and tumor resection. Although bone has a capacity for self-renewal, bone tissue regeneration remains a challenge because of its complex processes, including inflammation and bony callus formation [1]. To enhance bone growth, surgeons often use bone grafts or substitute materials [2]. In particular, bone autografting is clinically approved as the gold standard for bone repair because of the remarkable osteoinductivity and osteoconductivity without adverse immunoreactions
[3]. However, autogenous bone grafting has inevitable restrictions, including donor site morbidity, need for additional surgery, and limited bone donors [4, 5]. Therefore, promising strategies for bone defect reconstruction are required to overcome the obstacles and limitations in current bone grafting approaches. Research on bone repair has begun to focus on innovative tissue engineering technologies, as alternative approaches for functional tissue engineering [6].

In general, bone tissue engineering (BTE) begins with fabrication of a biocompatible scaffold, followed 
by its combination with cells and culture under specialized conditions that incorporate biochemical and physical stimuli to encourage bone formation in vitro and in vivo. Autologous bone marrow mesenchymal stem cells (BMMSCs) have been proposed as a suitable cell source for bone regeneration because of their lack of immunogenicity [7]. However, researchers have demonstrated that allogeneic mesenchymal stem cells maintained good cell viability without eliciting severe graft-versus-host disease [8, 9]. Li et al. [10] reported that rabbits showed immunological tolerance to green fluorescent protein-labeled allogeneic mesenchymal stem cells with no obvious rejection by the host. Thus, BMMSCs can be loaded into a scaffold and implanted in vivo without triggering an antigenic response. The primary purpose of biomaterials engineered for tissue regeneration is to support and facilitate the requisite physiological functions at the injured site. To satisfy this requirement, an ideal scaffold should possess favorable biocompatibility with optimal mechanical capabilities, and mimic a cell-friendly microenvironment that favors cell migration, proliferation, and differentiation [11, 12]. Nanohydroxyapatite (nHA) is a bioactive material that can mimic the nanostructure of natural bone as well as provide mechanical strength in the form of a scaffold. Furthermore, nHA was proven to have a significant influence on bone regeneration, through its formation of strong chemical bonds with the host bone tissue $[13,14]$. Gelatin (Gel), an important hydrocolloidal polypeptide, is produced by partial hydrolysis of collagen and facilitates initial cell adherence and spreading through its continuously repeated Arg-Gly-Asp (RGD) sequences. Gelatin microspheres (GMSs) have excellent biocompatibility and toxicologically safe degradation products, and have been widely selected as candidate carriers for sustained drug release to prolong the drug half-life and facilitate bone tissue regeneration [15-18]. To date, the prevailing approach in BTE has been combinations of scaffolds and osteogenic bioactive molecules important for promoting new bone formation and regulating cell behaviors like recruitment, proliferation, and differentiation. Growth factors, such as transforming growth factor- $\beta$, vascular endothelial growth factor, and bone morphogenetic proteins (BMPs), are signaling molecules and major factors that regulate cells during developmental processes. The BMP family and its individual members are regarded as crucial signaling proteins responsible for organization of tissue architecture. It is widely known that BMPs have significant roles in osteogenesis [19].
In the present study, we aimed to fabricate a BMP-6-nHA-Gel-GMS (BMP-6/nHAG/GMS) scaffold and evaluate its cytocompatibility and osteogenic activity in vitro. We also evaluated the in vivo bone regeneration efficacy of the scaffold using a critical-sized calvarial defect model in rats.

\section{Materials and methods}

\section{Materials}

Gelatin (Sigma-Aldrich, St. Louis, MO) and nHA (Emperor Nano Material, Nanjing, China) were chosen as the basic matrices for synthesis of the nHAG/GMS composite scaffold. Liquid paraffin (CAS\# 8042-47-5) was purchased from Aike Chemical Reagent Company (Chengdu, China). BMP-6 was purchased from PeproTech (Rocky Hill, NJ). Rat BMMSCs were purchased from PuheBio (Wuxi, China). Fetal bovine serum (FBS), phosphate-buffered saline (PBS), and alpha minimum essential medium (aMEM) were purchased from GE Healthcare Life Sciences Hyclone Laboratory (South Logan, Utah, USA). Penicillin/streptomycin and trypsin-EDTA were purchased from GE Healthcare Life Sciences Hyclone Laboratory (South Logan, UT). BMP-6 ELISA kits were purchased from Cusabio Biotechnology Company (Wuhan, China). Tetramethylrhodamine isothiocyanate (TRITC)-conjugated phalloidin was purchased from Invitrogen (Eugene, OR). Cell counting kit-8 (CCK-8) and alkaline phosphatase (ALP) kits were purchased from Nanjing Jiancheng Bioengineering Institute (Nanjing, China). All other chemicals and reagents were of analytical grade unless otherwise stated.

\section{Fabrication of porous BMP-6-loaded nHAG/GMS scaffolds}

GMSs were first prepared by an emulsion-solvent diffusion technique [20]. Briefly, a $25 \mathrm{wt} \%$ aqueous solution of gelatin was added dropwise to liquid paraffin, followed by stirring to obtain an emulsified compound. After the obtained compound was cooled to $4^{\circ} \mathrm{C}$, chilled acetone was then added, and GMSs were obtained after removal of the acetone. The obtained GMSs were crosslinked in glyoxal, washed with aqueous ethanol and dried. To fabricate the BMP-6/GMSs, BMP-6 was dissolved in PeproTech protein solution, and encapsulated in GMSs by adsorption and lyophilization.

For preparation of nHAG composite, nHA powder was homodispersed in a gelatin solution while stirring at $40^{\circ} \mathrm{C}$ [21]. The solution was poured into culture plates, frozen at $-20^{\circ} \mathrm{C}$ overnight, and lyophilized at $-80^{\circ} \mathrm{C}$ for $24 \mathrm{~h}$ using an Alpha 1-2 LD Plus (Christ, Germany). The resulting freeze-dried 
samples were immersed in glutaraldehyde aqueous solution for crosslinking, washed five times with deionized water, and freeze-dried again at $-80^{\circ} \mathrm{C}$.

For fabrication of BMP-6/nHAG/GMS scaffolds, the BMP-6/GMSs were dispersed in PBS and loaded in the nHAG composites by suction, resulting in a final BMP-6 concentration of $100 \mathrm{ng} / \mathrm{ml}$ in the BMP-6/nHAG/GMS scaffold.

\section{Scanning electron microscopy (SEM)}

The morphology of the nHAG/GMS scaffolds was examined by SEM (S-4800; Hitachi, Tokyo, Japan) after gold coating. The pore sizes were measured using Image J software.

\section{Porosity measurements}

The porosity of the nHAG/GMS scaffolds was evaluated by an ethyl alcohol $(\mathrm{EtOH})$ displacement method. The primary volume of EtOH was measured as V1. The scaffold was then immersed in a graduated cylinder containing EtOH until it reached saturation. During this process, trapped air was removed using a vacuum air-removal system. The total volume of EtOH and the scaffold was recorded as V2. The residual EtOH volume was measured as V3 after removal of the EtOH-impregnated scaffold. The porosity of the scaffold was calculated as: $[(\mathrm{V} 1-\mathrm{V} 3) /(\mathrm{V} 2-\mathrm{V} 3)] \times 100 \%$.

\section{Water absorption assay}

Water absorption was measured to assess the hydrophilic characteristics of the nHAG/GMS scaffolds. The dry scaffold was weighed (W1) and then immersed in distilled water until saturation. After blotting of excess water with filter paper, the scaffold was re-weighed (W2). The percentage of water absorption by the scaffold was calculated as: $[(\mathrm{W} 2-\mathrm{W} 1) / \mathrm{W} 1] \times 100 \%$.

\section{Mechanical properties}

The mechanical properties of the nHAG/GMS scaffolds were evaluated using a universal material testing machine (E1000; Instron, Norwood, MA). The diameter of the obtained scaffolds was $5.00 \mathrm{~mm}$, and the height was $10.00 \mathrm{~mm}$. Each sample was evaluated by application of a 100-N load at a crosshead speed of $1 \mathrm{~mm} / \mathrm{min}$. Three samples were examined to obtain the mean compression strength.

\section{In vitro release profile}

The in vitro BMP-6 release profile from the nHAG/GMS scaffolds was determined by ELISA. Briefly, the standard BMP-6/nHAG/GMS scaffold was incubated in a container containing $2 \mathrm{~mL}$ of PBS $(\mathrm{pH} 7.4)$ at $37^{\circ} \mathrm{C}$ in triplicate. At designated time points, the supernatant was collected for storage at $-20^{\circ} \mathrm{C}$ until analysis, and the sample was incubated in another $2 \mathrm{~mL}$ of fresh PBS. The cumulative release amount of BMP-6 was measured with the BMP-6 ELISA kit according to the manufacturer's procedure. The mean BMP-6 values were calculated and a release curve was drawn.

\section{Cell culture and seeding}

BMMSCs isolated from 3- to 4-week-old Sprague-Dawley rats were provided by PuheBio. BMMSCs were incubated in aMEM supplemented with $10 \% \quad(\mathrm{v} / \mathrm{v}) \quad$ FBS and $1 \% \quad(\mathrm{v} / \mathrm{v})$ penicillin/streptomycin and maintained at $37^{\circ} \mathrm{C}$ in a humidified $5 \% \quad \mathrm{CO}_{2}$ atmosphere. Scaffolds were sterilized with $75 \%$ (v/v) ethanol under UV light on both sides for $2 \mathrm{~h}$ each, and soaked in PBS for $2 \mathrm{~h}$ to favor scaffold wettability. The composite scaffolds were subsequently immersed in aMEM containing $10 \%$ FBS overnight at $37^{\circ} \mathrm{C}$. When cultured third-generation BMMSCs reached confluency, they were trypsinized and harvested. Next, $200 \mu \mathrm{L}$ of BMMSCs suspension $\left(4 \times 10^{4}\right.$ cells) was seeded into the sterilized scaffolds in 24-well culture plates to form cell-scaffold constructs. After $2 \mathrm{~h}$ of incubation, the culture plates were supplied with another $1 \mathrm{~mL}$ of culture medium. The specimens were cultured in vitro at $37^{\circ} \mathrm{C}$ in a humidified $5 \% \mathrm{CO}_{2}$ incubator, and the medium was changed every other day.

\section{Cell attachment and viability}

After 3 days of culture, the samples were examined by SEM to visualize the cell attachment. Briefly, the scaffolds with BMMSCs were gently rinsed twice with PBS, and fixed with $2.5 \%(\mathrm{w} / \mathrm{v})$ glutaraldehyde overnight at $4^{\circ} \mathrm{C}$. After washing with PBS, the samples were dehydrated in an ascending ethanol series at $30 \%, 50 \%, 70 \%, 80 \%, 90 \%$, and $100 \%$ for $20 \mathrm{~min}$ each. After complete drying, the samples were sputter-coated with gold and observed by SEM.

The cell-seeded scaffolds were cultured at $37^{\circ} \mathrm{C}$ in a humidified incubator with $5 \% \mathrm{CO}_{2}$ for 12 and 48 hours, mildly rinsed with PBS, and fixed with $4 \%$ (v/v) paraformaldehyde for $30 \mathrm{~min}$ at room temperature. After washing with PBS, the grafted cells were permeabilized with $0.2 \%(\mathrm{v} / \mathrm{v})$ Triton X-100 for $10 \mathrm{~min}$, and blocked with $1 \%(\mathrm{v} / \mathrm{v})$ BSA in PBS for $30 \mathrm{~min}$. The cytoskeletons of the cells were stained with TRITC-conjugated phalloidin for $2 \mathrm{~h}$ at $4^{\circ} \mathrm{C}$, and the nuclei were counter-stained with 4',6-diamidino-2-phenylindole (DAPI) for $10 \mathrm{~min}$ in the dark. The cytoskeletons and nuclei of cells were observed using a confocal laser scanning microscope (FV1000S-SIM/IX81; Olympus, Tokyo, Japan). After standard scanning processing, the fluorescence images were analyzed using Volocity Demo and Image Pro Plus 6.0 software. 


\section{Cell proliferation assay}

A direct contact method involving CCK-8 assays was applied to investigate the proliferation of BMMSCs on the composite scaffolds. Third-generation BMMSCs were seeded into the scaffolds with or without BMP-6. For the control group, BMMSCs were directly added to wells without scaffolds. After culture for 1, 3, 5, and 7 days, the medium was removed and $100 \mu \mathrm{L}$ of CCK-8 solution was added to each well and incubated for $4 \mathrm{~h}$. The culture solution $(300 \mu \mathrm{L})$ was then taken from the wells and transferred to a 96-well plate. The absorbances of the wells were measured by an ELISA assay reader (Infinite M200; Tecan, Austria) at $450 \mathrm{~nm}$.

\section{Alkaline phosphatase (ALP) activity}

After BMMSCs and composite scaffolds (nHAG/GMS and BMP-6/nHAG/GMS) were cocultured for 4,7 , and 10 days, three specimens per group were assessed for ALP activity according to the manufacturer's instructions. BMMSCs directly added into wells without any scaffolds were used as the control group. Briefly, the cells in the scaffolds were rinsed with PBS to remove the remaining medium, and immersed in $1 \%(\mathrm{v} / \mathrm{v})$ Triton-X 100 overnight at $4^{\circ} \mathrm{C}$. The cell suspension $(30 \mu \mathrm{L})$ was lysed by repeated pipetting and transferred to a 96-well Teflon culture plate. After adding buffer solution $(50 \mu \mathrm{L})$ and matrix liquid to the cell suspension, the mixture was incubated at $37^{\circ} \mathrm{C}$ for $15 \mathrm{~min}$. Each well was added with a chromogenic agent and the optical density (OD) was measured at $520 \mathrm{~nm}$ using the ELISA plate reader. The total cellular protein was measured by the bicinchoninic acid assay, and the ALP level was normalized by the total cellular protein content.

\section{Animals and anesthesia}

A total of 20 adult male Sprague-Dawley rats (8 weeks of age; 220-300 g) were provided by the
Experimental Animal Center of China Medical University. All in vivo animal experiments were reviewed and approved in advance by the Subcommittee on Research and Animal Care of China Medical University, and the procedures were carried out in strict accordance with the national guidelines for animal care. The rats were kept in plastic cages in an animal housing room that was maintained under standard laboratory facilities (12-h/12-h light/dark cycle; relative humidity: $45-55 \%$; temperature: $25^{\circ} \mathrm{C}$ ). All rats were acclimatized for at least 1 week, and provided with a standard laboratory diet and water. Surgical procedures were conducted under proper general anesthesia by intraperitoneal injection of $10 \%$ (v/v) chloral hydrate ( $3 \mathrm{~mL} / \mathrm{kg}$ body weight).

\section{Surgical procedure}

Prior to in vivo study, $4 \times 10^{4}$ rat BMMSCs were seeded on the sterilized scaffold sample and incubated for $24 \mathrm{~h}$ at $37^{\circ} \mathrm{C}$ in a humidified $5 \% \mathrm{CO}_{2}$ incubator. The rats were shaved and immobilized on a board that had been placed on a heating pad in advance. The surgical area was scrubbed with $10 \%$ $(\mathrm{v} / \mathrm{v})$ povidone iodine solution and $75 \%(\mathrm{v} / \mathrm{v})$ ethanol. A midline incision down to the periosteum was made using the scalpel and a full-thickness flap was elevated. After exposure of the calvarium, an 8-mm critical-sized defect was created using a trephine bur at low rotation under saline solution irrigation (Figure 1A and 1B). The 15 rats were randomly allocated to three groups: (1) no implantation group; (2) BMMSC/nHAG/GMS group; and (3) BMMSC/BMP-6/nHAG/GMS group. The periosteum was closed with a continuous suture before the incision was closed with 4-0 silk-interrupted sutures. The rats were housed for the designated time period according to the experimental protocol.
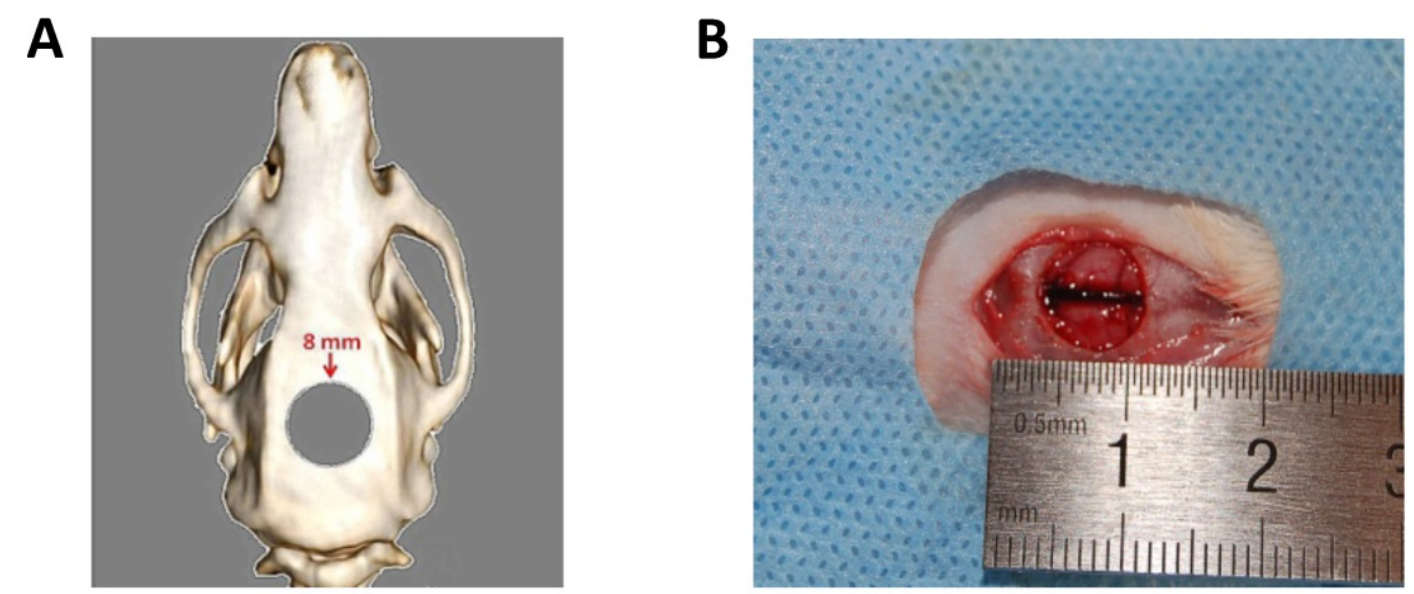

Figure 1. Preparation of critical-size calvarial bone defects. (A) Schematic drawing of rat calvarial defect. (B) Critical-sized defect with 8-mm diameter. 


\section{Postoperative examination and histological analysis}

At 8 weeks after implantation, all rats were euthanized by cervical dislocation under anesthesia with isoflurane. Three-dimensional images of the calvarial bones were taken using a 3D-CT scanner (SOMATOM Definition AS+; Siemens, Germany), and the CT values were measured to assess the density of regenerated tissue. After the 3D-CT examination, the skull caps were harvested and immediately fixed in $4 \%(\mathrm{v} / \mathrm{v})$ paraformaldehyde for histological analysis.

The calvarial bones were immersed in $10 \%(\mathrm{v} / \mathrm{v})$ EDTA solution for decalcification, and then dehydrated in a gradient alcohol series. After a final xylene step, the samples were embedded in paraffin. Serial sections at 5 - $\mu \mathrm{m}$ thickness were stained with hematoxylin and eosin (H\&E). The stained sections were observed and imaged by light microscopy (CKX41; Olympus Co., Tokyo, Japan), and the volumes of newly formed bone were measured using Image J software.

\section{Statistical analysis}

Statistical analyses were carried out with SPSS 17.0 software (SPSS Inc., Chicago, IL). All data were presented as mean \pm SD. Student's $t$-test was used for pairwise comparisons. Significance of differences in data was accepted for values of $p<0.05$.

\section{Results}

\section{Morphology and characterization of composite scaffolds}

The composite scaffolds were prepared by combination of a natural polymer, gelatin, and a bioceramic, nHA. After freeze-drying, the scaffolds exhibited a canary yellow color with good elasticity, and were able to return to their original state after compression deformation (Figure 2A).

The morphology and pore size distribution of the scaffolds were evaluated by SEM analysis. The micrographs revealed that the surface of the scaffolds was rough and uneven, with deposition of a large number of nHA particles. Furthermore, the structure had a three-dimensional architecture with interconnected pores of different sizes ranging from 100 to $200 \mu \mathrm{m}$ formed during freeze-drying (Figure 2B and $2 \mathrm{C}$ ).

By using the liquid displacement method, we obtained the porosity of the composite scaffolds was approximately $89 \%$. The water absorption capability was almost $258 \%$. During mechanical tests, the scaffolds exhibited good mechanical properties and the compressive strength was measured at $4.07 \mathrm{MPa}$ (Table 1).

\section{In vitro release of BMP-6}

The in vitro release profile of BMP-6 from the nHAG/GMS scaffolds was determined using an ELISA kit. The BMP-6-loaded scaffolds exhibited an initial burst release on day 1 and subsequently presented a gentler and constant release. Release of BMP-6 from the scaffold was continuously detected for 20 days and the cumulative release amount reached approximately 95\% (mean release: 92.15 $\pm 2.38 \%$ ) (Figure 2D).
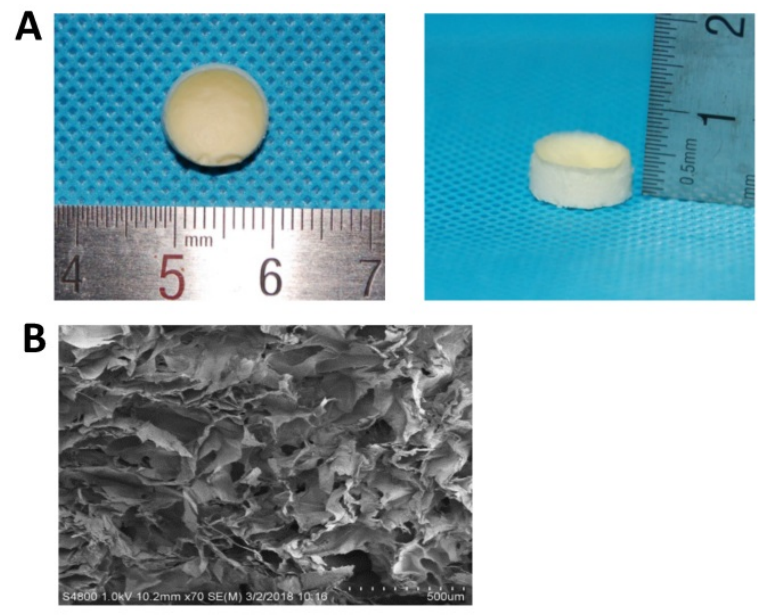

C

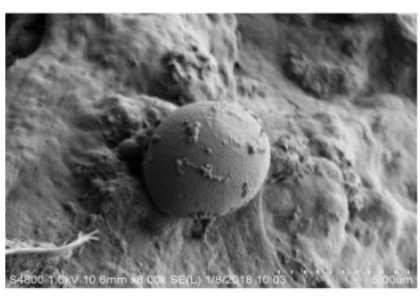

D

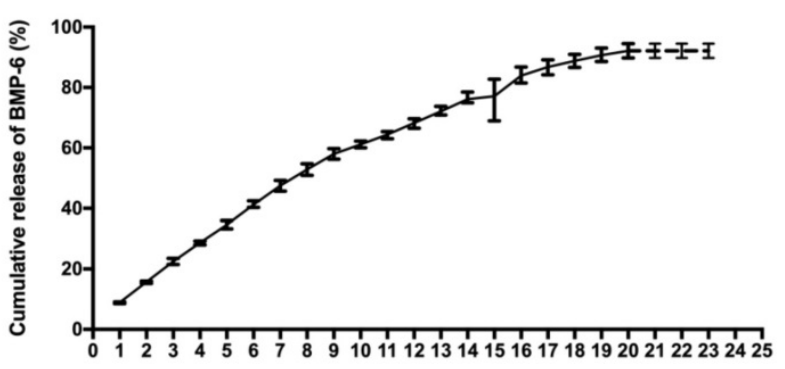

Figure 2. Structural characterization of the BMP-6/nHAG/GMS scaffold. (A) Macroscopic photograph of the BMP-6/nHAG/GMS scaffold. (B) SEM image of the BMP-6/nHAG/GMS scaffold. (C) Image of captured GMSs loaded onto the scaffold surface. (D) In vitro cumulative BMP-6 release profile from the BMP-6/nHAG/GMS scaffold over 20 days.

Table 1. Physical parameters of nHAG/GMS scaffolds

Porosity $(\%)$ Pore size $(\mu \mathrm{m}) \quad$ Water absorption (\%) Compressive strength $(\mathrm{MPa})$ $\begin{array}{llll}88.18 \pm 3.14 & 127 \pm 24 & 255.39 \pm 11.32 & 4.07 \pm 0.45\end{array}$

Results are presented as mean $\pm \mathrm{SD}(\mathrm{n}=3)$

\section{Cell morphology and viability}

The cellular morphology of rat BMMSCs was observed under an inverted phase-contrast 
microscope (CKX41; Olympus). Most of the cells adhered to the bottom of the culture plate within 4-8 $\mathrm{h}$ after passage. After $12 \mathrm{~h}$ of culture, BMMSCs exhibited a polygonal morphology and high survival rates during the culture period (Figure $3 \mathrm{~A}$ ).

To evaluate the BMMSC growth and attachment behavior on the scaffolds, which can indicate the possible impacts of composite materials, SEM cross-sectional observations were conducted at 3 days after BMMSC seeding. The micrographs revealed that abundant cells were strongly attached to the walls of the porous scaffolds with fully extended pseudopodia
(Figure 3B). In addition, the cells spread well and maintained their normal morphology.

To visualize the BMMSCs seeded on and within the three-dimensional scaffolds, the cell-scaffold constructs were stained with TRITC-conjugated phalloidin followed by nuclear staining with DAPI. Attachment of BMMSCs was observed at 12 hours after cell seeding on the scaffolds. The cells grew and proliferated well with an increased cell density on 48 hours of culture, and exhibited a clustered morphology with actin filaments linking adjacent cells (Figure 3C).

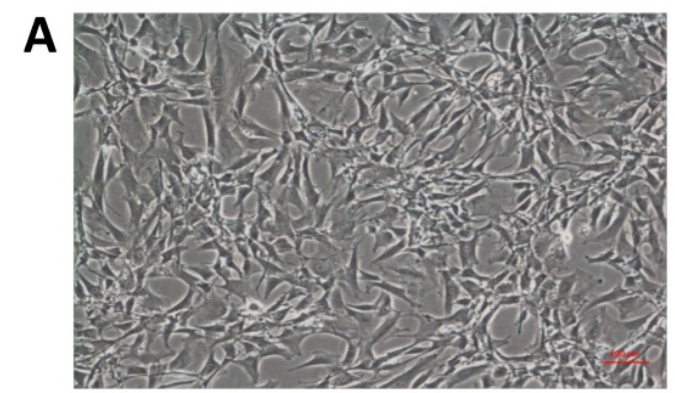

B
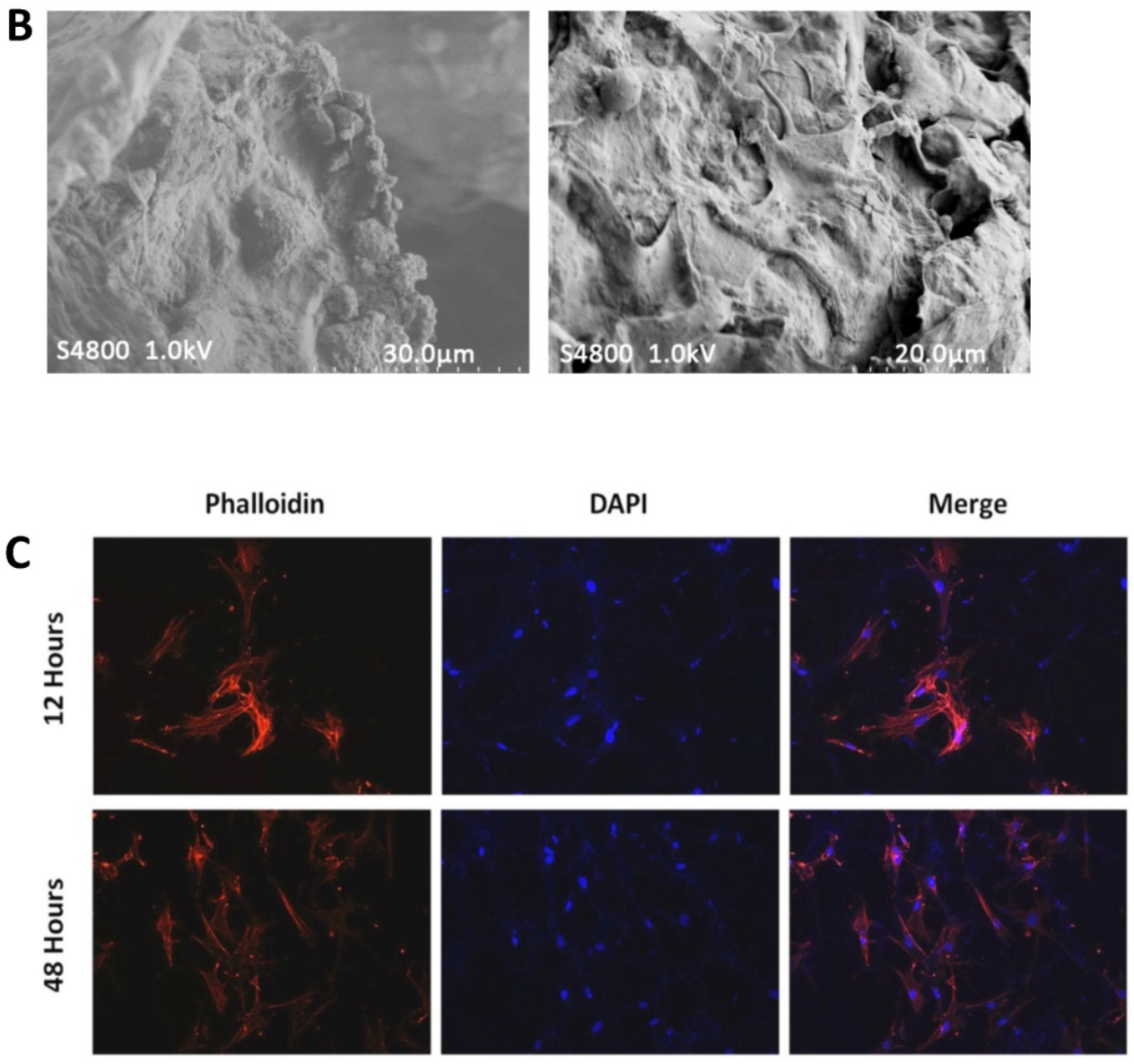

Figure 3. Cell morphology and viability. (A) Morphology of third-generation BMMSCs under an inverted phase-contrast microscope. (B) SEM images of cell-free (left) and cell-seeded (right) BMP-6/nHAG/GMS scaffolds. (C) In vitro fluorescence images of BMMSC attachment and proliferation on the BMP-6/nHAG/GMS scaffold at 12 and $48 \mathrm{~h}$. 

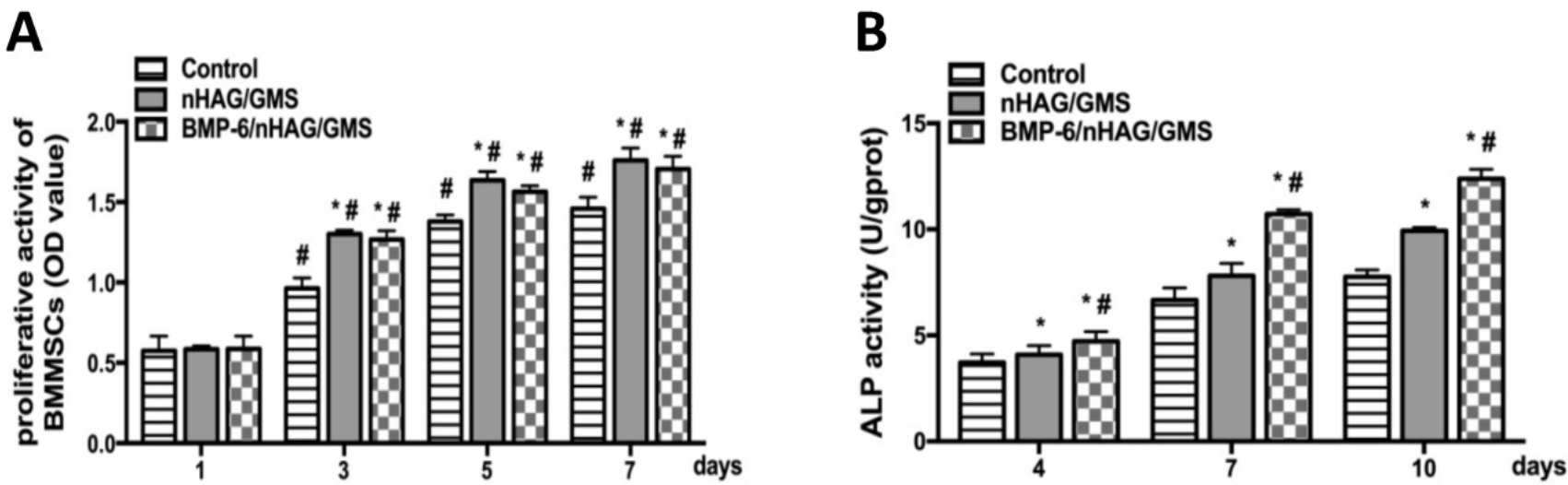

Figure 4. In vitro cell proliferation and osteogenesis. (A) CCK-8 assays for cell proliferation of BMMSCs. The nHAG/GMS and BMP-6/nHAG/GMS scaffolds show significantly accelerated BMMSC proliferation compared with the control group. ${ }^{*} \mathrm{P}<0.05$ vs. control group, $\# \mathrm{P}<0.05$ vs. previous time point of the same group. (B) ALP assays for osteogenic differentiation of BMMSCs. After 4 days, the ALP activity in the BMP-6/nHAG/GMS group is significantly enhanced compared with that in the $\mathrm{nHAG/GMS}$ and control groups. ${ }^{*} \mathrm{P}<0.05$ vs. control group, $\# \mathrm{P}<0.05$ vs. nHAG/GMS group.

\section{In vitro cell proliferation and osteogenesis}

To determine the effect of the scaffolds in supporting cell growth, CCK-8 assays were conducted to evaluate the OD values of the three groups on days 1, 3, 5, and 7 of culture. After 1 day of culture, there were no significant differences in cell proliferation among the three groups $(p>0.05)$, supporting the attachment and proliferation of BMMSCs. Starting from day 3, the nHAG/GMS and BMP-6/nHAG/GMS groups were more conducive to proliferation and showed significant differences compared with the control group $(p<0.05)$. However, there was no significant difference between the nHAG/GMS and BMP-6/nHAG/GMS groups $(p>0.05)$ (Figure 4A). The CCK-8 assay results indicated that the scaffolds had an appropriate capability to promote cell attachment and proliferation.

ALP activity, an early osteogenic differentiation marker, was assessed on days 4,7 , and 10 of culture to investigate the ability of the composite scaffolds to promote osteogenic differentiation of BMMSCs. No significant differences in the OD values were detected between the scaffold groups and the control group $(p>0.05)$ during the first 4 days of culture. Thereafter, the nHAG/GMS and BMP-6/nHAG/GMS groups induced remarkably higher ALP activity compared with the untreated control group $(p<0.05)$. Moreover, the OD value for the BMP-6-loaded nHAG/GMS scaffolds was superior to that of the nHAG/GMS scaffolds alone $(p<0.05)$, suggesting that the composite $3 \mathrm{D}$ scaffolds were capable of promoting osteogenic differentiation of BMMSCs in vitro (Figure 4B).

\section{In vivo repair efficacy of critical-sized calvarial defects}

All 15 rats tolerated the surgical operations, recovered well, and remained in good health through the experimental period. No signs of infection or postoperative wound-healing complications were observed at the defect site.

To evaluate new bone formation in vivo, the two types of porous composite scaffolds were implanted into the critical-sized calvarial defects, and 3D-CT analysis was conducted at week 8 postoperatively. On representative 3D-CT images, the defect-only group (control group) remained largely unrepaired with minimal bone regeneration at the edge, indicating that the critical-sized bone defects could not heal by themselves (Figure 5A). Meanwhile, the BMMSC/nHAG/GMS and BMMSC/BMP-6/nHAG/ GMS groups exhibited significantly smaller unhealed defect areas compared with the control group $(p<0.05)$. In addition, the BMP-6-loaded BMMSC/ nHAG/GMS scaffolds significantly accelerated new bone formation compared with the BMMSC/nHAG/ GMS group $(p<0.05)$, suggesting a function of BMP-6-modified nHAG/GMS scaffolds in inducing bone regeneration (Figure $5 \mathrm{~B}$ ).

Histomorphometric assessment of H\&E-stained sections confirmed the radiographic findings. The tissues in the defect-only group consisted of fibrous-like tissues with minimal bone formation at the margins with the host bone (Figure 6A). Compared with the control group, significantly larger bone formation areas and quantitative bone volumes were detected in the BMMSC/nHAG/GMS and BMMSC/BMP-6/nHAG/GMS groups $(p<0.05)$. Furthermore, the area of newly formed bone at the defect site was remarkably larger with the BMP-6-loaded composite scaffolds than with the BMP-6-free composite scaffolds $(p<0.05)$, in accordance with the bone volumes generated by the composite scaffolds (Figure 6B). 
A

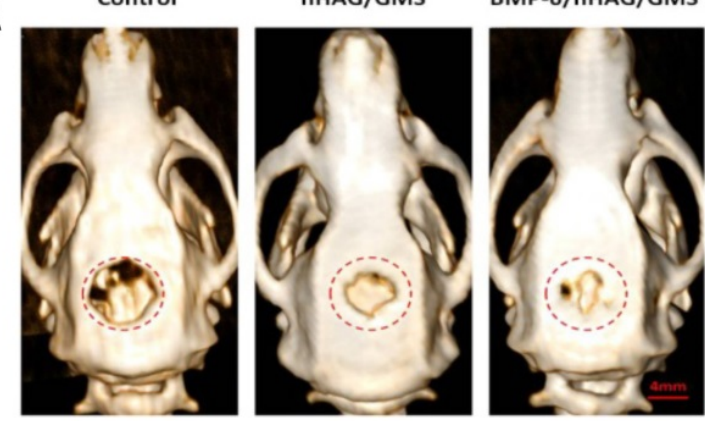

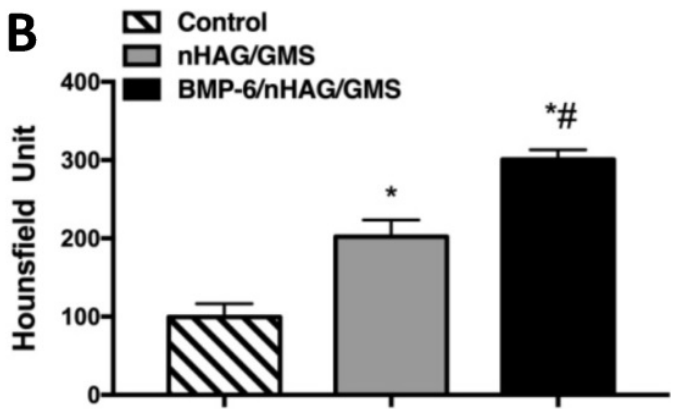

Figure 5. Analysis of calvarial defects at 8 weeks postoperatively. (A) 3D-CT determination of critical-sized defects after implantation with BMMSC-seeded nHAG/GMS and BMP-6/nHAG/GMS scaffolds compared with the control group. The dashed circles represent the original bony defects. (B) Quantitative measurements of bone formation in the control, BMMSC/nHAG/GMS, and BMMSC/BMP-6/nHAG/GMS groups. *P<0.05 vs. control group (defect-only group), \#P<0.05 vs. nHAG/GMS group.

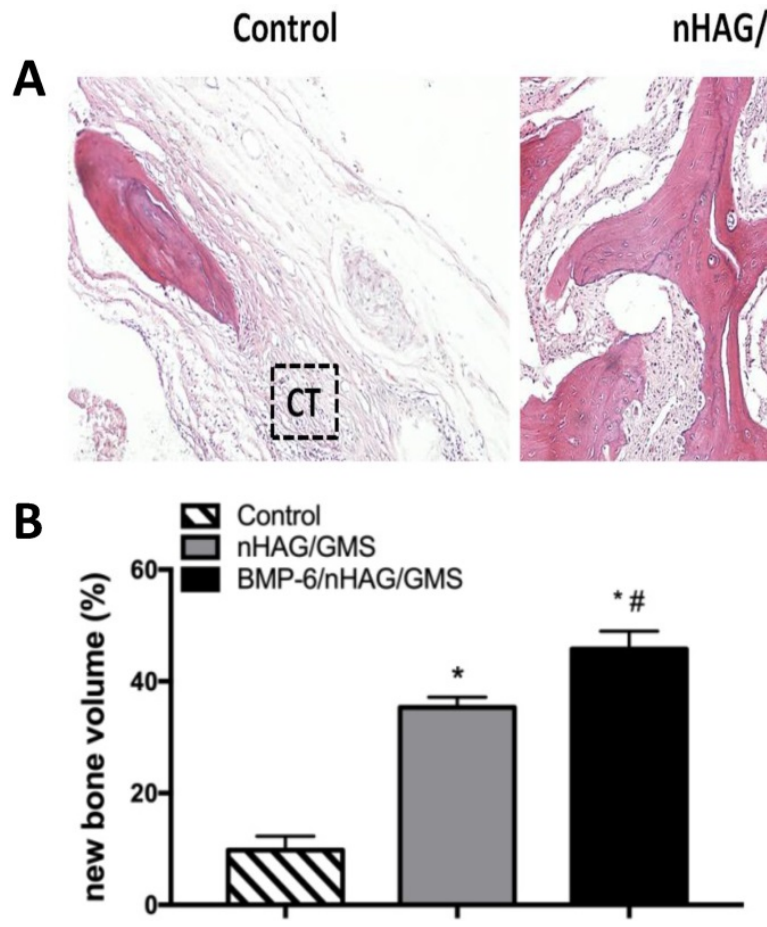

Figure 6. Histological and histomorphometric examinations at 8 weeks postoperatively. (A) Histological assessment of H\&E-stained sections in the three groups. (B) Histomorphometric measurements for evaluation of new bone formation areas. ${ }^{*} \mathrm{P}<0.05$ vs. control group (defect-only group), $\# \mathrm{P}<0.05$ vs. nHAG/GMS group.

\section{Discussion}

BTE is a complex process by which artificial organs are constructed using several factors, including viable cells, scaffolds, growth factors, and bio-conditions mimicking the in vivo microenvironment. Regarding scaffold design, achievement of an ideal scaffold that can integrate physical properties and biochemical cues is the way forward for next-generation development of bone tissue.

Natural bone is composed of inorganic salts and collagen. HA, a bioactive and biodegradable ceramic, has been widely applied as a bone graft substitute because of its superior osteoconductivity, osseointegration, and lack of toxicity [22]. nHA is the most abundant calcium phosphate mineral found in bone. Furthermore, owing to its small size and large specific surface area, nHA shows better performance in accelerating bone formation than traditional micro-sized ceramic materials [23-26]. Gelatin, which is derived from partial hydrolysis of collagen, has low immunogenicity and contains RGD-like sequences that favor cell migration and attachment [27]. Although natural biopolymers have the advantages of biodegradability and plasticity, their evident drawback of instability under physiological conditions cannot be ignored. In this regard, a composite scaffold that can combine nHA and a natural polymer to mimic the architecture of native bone to some extent has potential for bone regeneration.

In the present study, a nHAG/GMS scaffold was fabricated by a combination of blending and 
freeze-drying techniques, and sustained delivery of BMP-6 was obtained by encapsulation in GMSs. Subsequently, the features of the composite scaffold were evaluated in vitro and in vivo to investigate its potential to serve as an improved substitute for BTE purposes.

As reported in previous studies, the surface shape and texture of implants play important roles in modulating tissue reactions and cell activities [28, 29]. Furthermore, the rates of bone formation and vascularization were increased for implants prepared with interconnecting pores because of their adequate blood supply [30]. As shown in our SEM images, the surface of the BMP-6/nHAG/GMS scaffolds appeared to be rough because nHA particles were deposited over the surface. Wang et al. [31] reported that the pore sizes of an ideal scaffold should be greater than $100 \mu \mathrm{m}$, to facilitate bone mineralization and regeneration. The porosity and pore size of the obtained composite scaffolds exceeded $85 \%$ and 100 $\mu \mathrm{m}$, respectively, which can allow cells to infiltrate and fit well inside the scaffolds [32].

Biomimetic approaches have recently focused on combinations of bioactive scaffolds and growth factors with essential biological roles for bone regeneration. BMP-6 is regarded as a unique and crucial member of the BMP family for skeletal development. Previous studies revealed that BMP-6 was more efficient in inducing osteoblast differentiation of mesenchymal stem cells than BMP-2 and BMP-7, which were faced with issues of heterotopic ossification and early osteolysis [33-35]. To enhance growth factor delivery efficacy, microsphere-based sustained-release formulations have been used to improve the therapeutics of bone defects. Li et al. [36] described that vertebral formation was increased in osteoporotic animals after treatment with rhBMP-2/GMSs-loaded calcium phosphate cement (CPC), indicating that rhBMP-2/GMSs/CPC can facilitate bone healing in osteoporosis. As described in the present study, an nHAG scaffold integrated with BMP-6-loaded GMSs was applied and a series of measurements were performed to investigate the biocompatibility and osteogenic effect of the composite scaffold for bone regeneration. Reasonable density and uniform distribution of cells on scaffolds are key steps for three-dimensional culture [37]. According to the CCK-8 assay findings, the proliferation rates of BMMSCs in the composite scaffolds increased more quickly than those in the control group, which may due to the three-dimensional structure of the scaffolds. These findings are consistent with those of Kemençe et al. [38], who showed that gelatin- and hydroxyapatite-based cryogels crosslinked by glutaraldehyde did not decrease cell proliferation and viability in vitro. Moreover, the SEM and confocal laser scanning microscopy images revealed that the BMMSCs integrated in the 3D scaffolds protruded filopodia and stretched during extended culture duration, confirming that the scaffolds were conducive to cell adhesion and proliferation. ALP assessment and calvarial bone defect model creation were carried out to investigate the effects on osteogenesis. ALP activity is an accepted marker of osteogenic differentiation of cells and a higher level of ALP expression reflects a more differentiated phase [39-41]. The ALP determinations showed that the nHAG/GMS scaffolds could remarkably enhance BMMSC differentiation and that this effect was reinforced by incorporation of BMP- 6 within the scaffolds, indicating that the obtained scaffolds could effectively promote osteogenic differentiation in vitro. A critical-sized defect was originally defined as the smallest intraosseous wound in a particular bone and species of animal that cannot heal spontaneously during the lifetime of the animal [42]. It was reported that BMMSCs seeded on scaffolds can secrete a matrix that promotes new bone formation [43]. With this in mind, BMMSCs were seeded on nHAG/GMS and BMP-6/nHAG/GMS scaffolds, and then implanted into well-established critical-sized defects in rats. Murine models, which exhibit similar bone regenerative potential to humans, have been widely applied to determine the healing capacities of composite scaffolds [44]. Quinlan et al. [45] demonstrated that rhBMP-2-loaded collagenhydroxyapatite scaffolds effectively facilitated the healing of critical-sized defects at 8 weeks after implantation, with no apparent bone anomalies. In the present study, in vivo bone formation in critical-sized defects was detected by radiographic and histological analyses at 8 weeks after implantation. Compared with the other groups, the BMMSC-seeded BMP-6/nHAG/GMS group exhibited significantly augmented in vivo calvarial bone formation, thereby verifying that use of the BMP-6/nHAG/GMS scaffolds is an effective approach for bone regeneration. In addition, it is worth noting that the periosteum and dura mater should be carefully preserved for better bone regeneration. The beneficial functions of the periosteum and dura mater noted here are consistent with previous studies [46, 47].

In conclusion, a porous BMP-6-loaded nHAG/GMS scaffold was prepared by utilizing a combination of blending and freeze-drying techniques. In vitro measurements confirmed that the scaffold was biocompatible with ideal characteristics and was able to induce osteogenic differentiation. In 
vivo assays verified that the BMMSC-seeded BMP-6/nHAG/GMS scaffold could successfully accelerate new bone formation. Accordingly, use of the BMP-6/nHAG/GMS scaffold could be a promising approach for BTE.

\section{Abbreviations}

BTE: bone tissue engineering; BMMSCs: bone marrow mesenchymal stem cells; nHA: nanoHydroxyapatite; Gel: gelatin; GMSs: gelatin microspheres; BMP-6: bone morphogenetic protein 6; nHAG/GMS: nHA/Gel/GMS scaffold; BMP-6/ nHAG/GMS: BMP-6/nHA/Gel/GMS scaffold; FBS: Fetal bovine serum; PBS: phosphate-buffered saline; aMEM: alpha minimum essential medium; SEM: scanning electron microscope; EtOH: ethyl alcohol; CCK-8: cell counting kit-8; ALP: alkaline phosphatase.

\section{Acknowledgments}

This work was financially supported by National Natural Science Foundation of China (Grant No. 81600825), Natural Science Foundation of Liaoning Province of China (Grant No. 201602856) and Science \& Technology Foundation of Shenyang (Grant No. 17-231-1-50).

\section{Competing Interests}

The authors have declared that no competing interest exists.

\section{References}

1. Kim YH, Tabata Y. Dual-controlled release system of drugs for bone regeneration. Adv Drug Deliv Rev. 2015; 94: 28-40.

2. Sharma C, Dinda AK, Potdar PD, et al. Fabrication and characterization of novel nano-biocomposite scaffold of chitosan-gelatin-alginatehydroxyapatite for bone tissue engineering. Mater Sci Eng C Mater Biol Appl. 2016; 64: 416-27.

3. Zhang J, Liu W, Schnitzler V, et al. Calcium phosphate cements for bone substitution: chemistry, handling and mechanical properties. Acta Biomater. 2014; 10 : 1035-49.

4. Farokhi M, Mottaghitalab F, Shokrgozar MA, et al. Importance of dual delivery systems for bone tissue engineering. J Control Release. 2016; 225: 152-69.

5. Fernandez-Yague MA, Abbah SA, McNamara L, et al. Biomimetic approaches in bone tissue engineering: Integrating biological and physicomechanical strategies. Adv Drug Deliv Rev. 2015; 84:1-29.

6. Lickorish D, Guan L, Davies JE. A three-phase, fully resorbable, polyester/calcium phosphate scaffold for bone tissue engineering: evolution of scaffold design. Biomaterials. 2007; 28: 1495-502

7. Vilquin JT, Rosset P. Mesenchymal stem cells in bone and cartilage repair: current status. Regen Med. 2006; 1: 589-604.

8. Liu Z, Yuan X, Fernandes G, et al. The combination of nano-calcium sulfate/platelet rich plasma gel scaffold with BMP2 gene-modified mesenchymal stem cells promotes bone regeneration in rat critical-sized calvarial defects. Stem Cell Res Ther. 2017; 8: 122

9. Saito T, Kuang JQ, Bittira B, et al. Xenotransplant cardiac chimera: immune tolerance of adult stem cells. Ann Thorac Surg. 2002; 74: 19-24. Discussion 24.

10. $\mathrm{Li} \mathrm{ZH}$, Liao $\mathrm{W}$, Cui $\mathrm{XL}$, et al. Intravenous transplantation of allogeneic bone marrow mesenchymal stem cells and its directional migration to the necrotic femoral head. Int J Med Sci. 2011; 8: 74-83.

11. Hollister SJ. Porous scaffold design for tissue engineering. Nat Mater. 2005; 4: 518-24.

12. Ou KL, Hosseinkhani H. Development of 3D in vitro technology for medical applications. Int J Mol Sci. 2014; 15: 17938-62.

13. Baroli B. From natural bone grafts to tissue engineering therapeutics: Brainstorming on pharmaceutical formulative requirements and challenges. J Pharm Sci. 2009; 98: 1317-75.
14. Mehedi Hasan M, Nuruzzaman Khan $M$, Haque $P$, et al. Novel alginate-di-aldehyde cross-linked gelatin/nano-hydroxyapatite bioscaffolds for soft tissue regeneration. Int J Biol Macromol. 2018; 117: 1110-17.

15. Yan J, Miao Y, Tan $\mathrm{H}$, et al. Injectable alginate/hydroxyapatite gel scaffold combined with gelatin microspheres for drug delivery and bone tissue engineering. Mater Sci Eng C Mater Biol Appl. 2016; 63: 274-84.

16. Barbetta A, Dentini M, Zannoni EM, et al. Tailoring the porosity and morphology of gelatin-methacrylate polyHIPE scaffolds for tissue engineering applications. Langmuir. 2005; 21: 12333-41.

17. Vandelli MA, Romagnoli M, Monti A, et al. Microwave-treated gelatin microspheres as drug delivery system. J Control Release. 2004; 16: 67-84.

18. Aviv-Gavriel M, Garti N, Füredi-Milhofer H. Preparation of a Partially Calcified Gelatin Membrane as a Model for a Soft-to-Hard Tissue Interface. Langmuir 2013; 29: 683-9.

19. Mizrahi O, Sheyn D, Tawackoli W, et al. BMP-6 is more efficient in bone formation than BMP-2 when overexpressed in mesenchymal stem cells. Gene Ther. 2013; 20: 370-7

20. Holland TA, Tabata Y, Mikos AG. In vitro release of transforming growth factor-beta 1 from gelatin microparticles encapsulated in biodegradable, injectable oligo (poly (ethylene glycol) fumarate) hydrogels. J Control Release. 2002; 19: 363-76

21. Azami M, Samadikuchaksaraei A, Poursamar SA. Synthesis and characterization of a laminated hydroxyapatite/gelatin nanocomposite scaffold with controlled pore structure for bone tissue engineering. Int J Artif Organs. 2010; 33: 86-95.

22. Szcześ A, Holysz L, Chibowski E. Synthesis of hydroxyapatite for biomedical applications. Adv Colloid Interface Sci. 2017; 249: 321-30.

23. Kubasiewicz-Ross P, Hadzik J, Seeliger J, et al. New nano-hydroxyapatite in bone defect regeneration: a histological study in rats. Ann Anat. 2017; 213: 83-90.

24. Ao C, Niu Y, Zhang X, et al. Fabrication and characterization of electrospun cellulose/nano-hydroxyapatite nanofibers for bone tissue engineering. Int J Biol Macromol. 2017; 97: 568-73.

25. Atak BH, Buyuk B, Huysal M, et al. Preparation and characterization of amine functional nano-hydroxyapatite/chitosan bionanocomposite for bone tissue engineering applications. Carbohydr Polym. 2017; 164: 200-13.

26. Forero JC, Roa E, Reyes JG, et al. Development of Useful Biomaterial for Bone Tissue Engineering by Incorporating Nano-Copper-Zinc Alloy (nCuZn) in Chitosan/Gelatin/NanoHydroxyapatite (Ch/G/nHAp) Scaffold. Materials (Basel). 2017; 10: 1177.

27. Schönwälder SM, Bally F, Heinke L, et al. Interaction of human plasma proteins with thin gelatin-based hydrogel films: A QCM-D and ToF-SIMS study. Biomacromolecules. 2014; 15: 2398-406.

28. Guillaume O, Geven MA, Sprecher $\mathrm{CM}$, et al. Surface-enrichment with hydroxyapatite nanoparticles in stereolithography-fabricated composite polymer scaffolds promotes bone repair. Acta Biomater. 2017; 54: 386-98.

29. Zeng S, Ye J, Cui Z, et al. Surface biofunctionalization of three-demensional porous poly (lactic acid) scaffold using chitosan/OGP coating for bone tissue engineering. Mater Sci Eng C Mater Biol Appl. 2017; 77: 92-101.

30. Bose S, Vahabzadeh S, Bandyopadhyay A. Bone tissue engineering using 3D printing. Materialstoday. 2013; 6: 496-504.

31. Wang $\mathrm{X}, \mathrm{Li} \mathrm{J}, \mathrm{Hu} \mathrm{R}$, et al. Mechanical properties of porous titanium with different distributions of pore size. Trans Nonferrous Met Soc. 2013; 23: 2317-22.

32. Roohani-Esfahani SI, No YJ, Lu Z et al A bioceramic with enhanced osteogenic properties to regulate the function of osteoblastic and osteocalastic cells for bone tissue regeneration. Biomed Mater. 2016; 11: 035018.

33. Lissenberg-Thunnissen SN, de Gorter DJ, Sier CF, et al. Use and efficacy of bone morphogenetic proteins in fracture healing. Int Orthop. 2011; 35: 1271-80.

34. Ji W, Kerckhofs G, Geeroms C, et al. Deciphering the combined effect of bone morphogenetic protein 6 and calcium phosphate on bone formation capacity of periosteum derived cells-based tissue engineering constructs. Acta Biomater. 2018; 80: 97-107.

35. Vukicevic S, Oppermann H, Verbanac D, et al. The clinical use of bone morphogenetic proteins revisited: a novel biocompatible carrier device OSTEOGROW for bone healing. Int Orthop. 2014; 38: 635-47.

36. Li M, Liu X, Liu X, et al. Calcium phosphate cement with BMP-2-loaded gelatin microspheres enhances bone healing in osteoporosis. Clin Orthop Relat Res. 2010; 468: 1978-85.

37. Issa RI, Engebretson B, Rustom L, et al. The effect of seeding density on the cellular and mechanical properties of a mechanostimulated tissue-engineered tendon. Tissue Eng Part A. 2011; 17: 1479-87.

38. Kemençe N, Bölgen N. Gelatin- and hydroxyapatite-based cryogels for bone tissue engineering: synthesis, characterization, in vitro and in vivo biocompatibility. J Tissue Eng Regen Med. 2017; 11: 20-33.

39. Cho HJ, Perikamana SK, Lee JH, et al. Effective immobilization of BMP-2 mediated by polydopamine coating on biodegradable nanofibers for enhanced in vivo bone formation. ACS Appl Mater Interfaces. 2014; 6: 11225-35.

40. Ehara A, Ogata K, Imazato S, et al. Effects of a-TCP and TetCP on MC3T3-E1 proliferation, differentiation and mineralization. Biomaterials. 2003; 24: 831-6.

41. Zhou J, Wei F, Ma Y. Inhibiting PPARY by erythropoietin while upregulating TAZ by IGF1 synergistically promote osteogenic differentiation of mesenchymal stem cells. Biochem Biophys Res Commun. 2016; 478: 349-55. 
42. Li J, Jahr H, Zheng $\mathrm{W}$, et al. Visualizing angiogenesis by multiphoton microscopy in vivo in genetically modified 3D-PLGA/nHAp scaffold for calvarial critical bone defect repair. J Vis Exp. 2017; 127: 1-10.

43. Hamidabadi HG, Shafaroudi MM, Seifi M, et al. Repair of critical-sized rat calvarial defects with three-dimensional hydroxyapatite-gelatin scaffolds and bone marrow stromal stem cells. Med Arch. 2018; 72: 88-93.

44. Kim EC, Kim TH, Jung JH, et al. Enhanced osteogenic differentiation of MC3T3-E1 on rhBMP-2-immobilized titanium via click reaction. Carbohydr Polym. 2014; 103: 170-8.

45. Quinlan E, Thompson EM, Matsiko A, et al. Long-term controlled delivery of rhBMP-2 from collagen-hydroxyapatite scaffolds for superior bone tissue regeneration. J Control Release. 2015; 207: 112-9.

46. Liu W, Kang N, Dong Y, et al. Effect of resorbable collagen plug on bone regeneration in rat critical-size defect model. Implant Dent. 2016; 25: 163-70.

47. Spicer PP, Kretlow JD, Young S, et al. Evaluation of bone regeneration using the rat critical size calvarial defect. Nat Protoc. 2017; 7: 1918-29. 